\title{
An acute coronary event due to vasospasm in a young healthy male with normal epicardial vessels
}

\author{
Seneviratne T S $\mathbf{P}^{1}$, Kumanan $\mathbf{T}^{1}$, Logeswaran $\mathbf{D}^{1}$, Guruparan $\mathbf{M}^{1}$ \\ Journal of the Ceylon College of Physicians, 2016, 47, 48-49
}

\section{Introduction}

Coronary artery spasm is shown to play an important role in the pathogenesis of myocardial infarction, with or without significant coronary artery stenosis. Here we report a rare occurrence of acute coronary ischaemia that occurred few minutes after trauma, in a patient who was previously healthy and had no symptoms suggestive of coronary artery disease.

\section{Case report}

An 18-year-old school boy was admitted to emergency department for left sided chest pain following trauma. He had several deep cut injuries over his left forearm and scalp as well as a superficial cut injury over left lateral chest. He had a typical ischaemic type of chest pain after the incident. The pain lasted only about a minute. It was associated with palpitations. A similar, less intense episode of pain followed a few minutes later. Except for the cut injuries his physical examination was unremarkable. The ECG showed T inversions in V3-V6 (Figure 1). Troponin I was positive with a value of 4.09 . The chest radiograph and 2-D echocardiography were normal. With the history of classical anginal chest pain, positive troponin and dynamic ECG changes, a diagnosis of non ST elevation myocardial infarction was made.

He was treated with dual antiplatelet drugs, statins and enoxaparin for 3 days. The ECG reverted to normal within the next 48 hours and the troponin I levels gradually came down over the next few days. The coronary angiogram performed on Day 4 showed normal epicardial coronary vessels.

As the history, clinical features, laboratory investigations and ECG strongly suggestive of an acute

\footnotetext{
${ }^{1}$ Department of Medicine, University of Jaffna, Teaching Hospital, Jaffna.
}

coronary event, in the context of normal coronary vasculature, the most probable cause for the ischemic event would have been the stress induced vasospasm of coronary arteries.

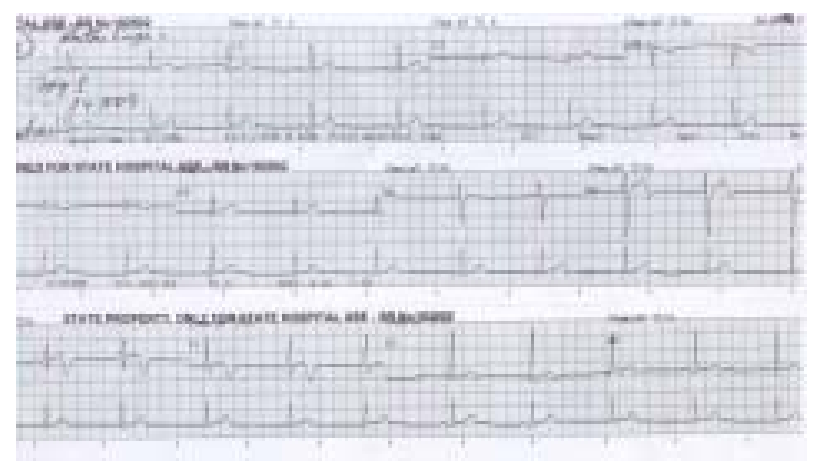

Figure 1. $\mathrm{T}$ inversions in leads V3-V6.

\section{Discussion}

Although coronary artery atherosclerosis is the most common cause of acute myocardial infarction, $20 \%$ of acute myocardial infarction in young adults have non-atherosclerotic aetiology such as coronary artery embolism, hypercoagulation status, congenital coronary abnormalities, dissection of coronary arteries, vasculitis and coronary artery spasm. ${ }^{1}$ The mechanism proposed to constitute susceptibility to coronary artery spasm include, endothelial dysfunction, primary hyperreactivity of vascular smooth muscle and other factors. ${ }^{2}$

The stimuli that trigger Coronary artery spasm are still incompletely understood, and may not be readily identifiable in individual patients. However several vasoconstrictor stimuli are likely to trigger the spasm in the same patient when acting at the site of hyper-reactive coronary segment. ${ }^{2}$ Among the potential triggers of coronary artery spasm, the autonomic nervous system has received a great deal of attention. The relationship between autonomic nervous system and coronary artery spasm, however, is rather complex, because both increase in sympathetic tone and increase in parasympathetic tone seem to induce 
coronary artery spasm. ${ }^{3}$ Noradrenaline, the neuro transmitter of efferent sympathetic fibers can trigger vasoconstriction in vascular smooth muscle through stimulation of alpha adrenergic receptors. Clinical studies have confirmed that coronary artery vasospasm can be induced by catecholamine. ${ }^{3}$ An angiotensin II type 1 receptor gene polymorphism has been shown to be associated with increased tendency to vasospasm in angiographically normal coronary arteries challenged with vasoconstrictors. ${ }^{4}$

In our patient it seems coronary artery vasospasm was the cause of myocardial infarction without obstructive coronary artery disease.

\section{References}

1. Andishmand A, Banifatemeh SA, Behnamfar Z, Mirvakili SM. Acute myocardial infarction with normal coronary artery following trauma. Journal of Biology and Today's World 2014; 3(9): 198-200.

2. Stern S, de LunaAB. Coronary artery spasm: A2009 Update. Circulation 2009; 119: 2531-4.

3. Lanza GA, Careri G, Crea F. Mechanisms of coronary artery spasm. Circulation 2011; 124: 1774-82.

4. Kardasz R, Caterina RD. Myocardial infarction with normal coronary arteries: a conundrum with multiple aetiologies and variable prognosis: an update. Journal of Internal Medicine 2007; 261; 330-48. 